Pure and Applied Mathematics Quarterly

Volume 8, Number 2

(Special Issue: In honor of

F. Thomas Farrell and Lowell E. Jones, Part 2 of 2)

$313-327,2012$

\title{
The Atiyah Conjecture and Artinian Rings
}

\author{
Peter A. Linnell and Thomas Schick
}

\begin{abstract}
Let $G$ be a group such that its finite subgroups have bounded order, let $d$ denote the lowest common multiple of the orders of the finite subgroups of $G$, and let $K$ be a subfield of $\mathbb{C}$ that is closed under complex conjugation. Let $\mathcal{U}(G)$ denote the algebra of unbounded operators affiliated to the group von Neumann algebra $\mathcal{N}(G)$, and let $\mathcal{D}(K G, \mathcal{U}(G))$ denote the division closure of $K G$ in $\mathcal{U}(G)$; thus $\mathcal{D}(K G, \mathcal{U}(G))$ is the smallest subring of $\mathcal{U}(G)$ containing $K G$ that is closed under taking inverses. Suppose $n$ is a positive integer, and $\alpha \in \mathrm{M}_{n}(K G)$. Then $\alpha$ induces a bounded linear map $\alpha: \ell^{2}(G)^{n} \rightarrow \ell^{2}(G)^{n}$, and ker $\alpha$ has a well-defined von Neumann dimension $\operatorname{dim}_{\mathcal{N}(G)}(\operatorname{ker} \alpha)$. This is a nonnegative real number, and one version of the Atiyah conjecture states that $d \operatorname{dim}_{\mathcal{N}(G)}(\operatorname{ker} \alpha) \in \mathbb{Z}$. Assuming this conjecture, we shall prove that if $G$ has no nontrivial finite normal subgroup, then $\mathcal{D}(K G, \mathcal{U}(G))$ is a $d \times d$ matrix ring over a skew field. We shall also consider the case when $G$ has a nontrivial finite normal subgroup, and other subrings of $\mathcal{U}(G)$ that contain $K G$.
\end{abstract}

Keywords: Atiyah conjecture, group von Neumann algebra.

\section{INTRODUCTION}

In this paper $\mathbb{N}$ will denote the positive integers $\{1,2, \ldots\}$, all rings will have a 1 , subrings will have the same 1 , and if $n \in \mathbb{N}$, then $\mathrm{M}_{n}(R)$ will indicate the $n \times n$ matrices over the ring $R$ and $\mathrm{GL}_{n}(R)$ the invertible matrices in $\mathrm{M}_{n}(R)$.

Received November 22, 2007.

2000 Mathematics Subject Classification. Primary: 16S34; Secondary: 20C07, 22D25, 46L99. 
Let $G$ be a group, let $\ell^{2}(G)$ denote the Hilbert space with orthonormal basis the elements of $G$, and let $\mathcal{B}\left(\ell^{2}(G)\right)$ denote the bounded linear operators on $\ell^{2}(G)$. Thus we can write elements $a \in \ell^{2}(G)$ in the form $\sum_{g \in G} a_{g} g$, where $a_{g} \in \mathbb{C}$ and $\sum_{g \in G}\left|a_{g}\right|^{2}<\infty$. Then $\mathbb{C} G$ acts faithfully on the left of $\ell^{2}(G)$ as bounded linear operators via the left regular representation, so we may consider $\mathbb{C} G$ as a subalgebra of $\mathcal{B}\left(\ell^{2}(G)\right)$. The weak closure of $\mathbb{C} G$ in $\mathcal{B}\left(\ell^{2}(G)\right)$ is the group von Neumann algebra $\mathcal{N}(G)$ of $G$. Also if $n \in \mathbb{N}$, then $\mathrm{M}_{n}(\mathbb{C} G)$ acts as bounded linear operators on $\ell^{2}(G)^{n}$ and the weak closure of this ring in $\mathcal{B}\left(\ell^{2}(G)^{n}\right)$ is $\mathrm{M}_{n}(\mathcal{N}(G))$. Let 1 indicate the element of $\ell^{2}(G)$ which is 1 at the identity of $G$ and zero elsewhere. Then the map $\theta \mapsto \theta 1: \mathcal{N}(G) \rightarrow \ell^{2}(G)$ is an injection, so we may regard $\mathcal{N}(G)$ as a subspace of $\ell^{2}(G)$. We can now define $\operatorname{tr}: \mathcal{N}(G) \rightarrow \mathbb{C}$ by $\operatorname{tr}(a)=a_{1}$. For $\alpha \in \mathrm{M}_{n}(\mathcal{N}(G))$, we can extend this definition by setting $\operatorname{tr}(\alpha)=\sum_{i=1}^{n} \operatorname{tr}\left(\alpha_{i i}\right)$, where $\alpha_{i j}$ are the entries of $\alpha$. A useful property is that if $\alpha$ is a positive operator, then $\operatorname{tr}(\alpha) \geq 0$. Also we can use tr to give any right $\mathcal{N}(G)$-module $M$ a well defined dimension $\operatorname{dim}_{\mathcal{N}(G)} M$, which in general is a non-negative real number or $\infty[10, \S 6.1]$. If $e$ is a projection in $\mathrm{M}_{n}(\mathcal{N}(G))$, then $\operatorname{dim}_{\mathcal{N}(G)} e \mathrm{M}_{n}(\mathcal{N}(G))=\operatorname{tr}(e)$. Furthermore if $\alpha \in \mathrm{M}_{n}(\mathcal{N}(G))$, so $\alpha$ is a Hilbert space map $\ell^{2}(G)^{n} \rightarrow \ell^{2}(G)^{n}$, then since $\ell^{2}(G)^{n}$ is a right $\mathcal{N}(G)$-module, $\operatorname{dim}_{\mathcal{N}(G)} \operatorname{ker} \alpha$ is well defined and is equal to $\operatorname{dim}_{\mathcal{N}(G)}\left\{\beta \in \mathcal{N}(G)^{n} \mid \alpha \beta=0\right\}$. Finally $\mathcal{N}(G)$ has an involution which sends an operator to its adjoint; if $a=$ $\sum_{g \in G} a_{g} g$, then $a^{*}=\sum_{g \in G} \bar{a}_{g} g^{-1}$, where the bar indicates complex conjugation.

A ring $R$ is called regular, or sometimes von Neumann regular, if for every $x \in R$, there exists an idempotent $e \in R$ with $x R=e R$ [5, Theorem 1.1]. It is called finite, or directly finite, if $x y=1$ implies $y x=1$ for all $x, y \in R$. Finally a $*$-regular ring $R$ is a regular ring with an involution * with the property that $x \in R$ and $x^{*} x=0$ implies $x=0$. In a $*$-regular ring, given $x \in R$, there is a unique projection $e$ such that $x R=e R$; so $e=e^{*}=e^{2}$.

Let $\mathcal{U}(G)$ denote the algebra of unbounded operators on $\ell^{2}(G)$ affiliated to $\mathcal{N}(G)[10, \S 8]$. Then the involution on $\mathcal{N}(G)$ extends to an involution on $\mathcal{U}(G)$, and $\mathcal{U}(G)$ is a finite $*$-regular algebra. Also if $M$ is a right $\mathcal{N}(G)$-module, then $\operatorname{dim}_{\mathcal{N}(G)} M=\operatorname{dim}_{\mathcal{N}(G)} M \otimes_{\mathcal{N}(G)} \mathcal{U}(G)$; in particular $\operatorname{dim}_{\mathcal{N}(G)} e \mathcal{U}(G)=\operatorname{tr}(e)$.

For any subring $R$ of the ring $S$, we let $\mathcal{D}(R, S)$ denote the division closure of $R$ in $S$; that is the smallest subring of $S$ containing $R$ that is closed under taking inverses. In the case $G$ is a group and $K$ is a subfield of $\mathbb{C}$, we shall set 
$\mathcal{D}(K G)=\mathcal{D}(K G, \mathcal{U}(G))$. For any group $G$, let $\operatorname{lcm}(G)$ indicate the least common multiple of the orders of the finite subgroups of $G$, and adopt the convention that $\operatorname{lcm}(G)=\infty$ if the orders of the finite subgroups of $G$ are unbounded. One version of the strong Atiyah conjecture states that if $G$ is a group with $\operatorname{lcm}(G)<\infty$, then the $L^{2}$-Betti numbers of every closed manifold with fundamental group $G$ lie in the abelian group $\frac{1}{\operatorname{lcm}(G)} \mathbb{Z}$. This is equivalent to the conjecture that if $n \in \mathbb{N}$, $A \in \mathrm{M}_{n}(\mathbb{Q} G)$ and $\alpha: \ell^{2}(G)^{n} \rightarrow \ell^{2}(G)^{n}$ is the map induced by left multiplication by $A$, then $\operatorname{lcm}(G) \operatorname{dim}_{\mathcal{N}(G)} \operatorname{ker} \alpha \in \mathbb{Z}[9$, Lemma 2.2]. In this paper, we shall consider more generally the case when the coefficient ring is a subfield of $\mathbb{C}$.

Definition 1.1. Let $G$ be a group with $\operatorname{lcm}(G)<\infty$, and let $K$ be a subfield of $\mathbb{C}$. We say that the strong Atiyah conjecture holds for $G$ over $K$ if

$$
\operatorname{lcm}(G) \operatorname{dim}_{\mathcal{N}(G)} \operatorname{ker} \alpha \in \mathbb{Z} \quad \text { for all } \alpha \in \mathrm{M}_{n}(K G) .
$$

This is equivalent to the conjecture that if $M$ is a finitely presented $K G$ module, then $\operatorname{lcm}(G) \operatorname{dim}_{\mathcal{N}(G)} M \otimes_{K G} \mathcal{N}(G) \in \mathbb{Z}[10$, Lemma 10.7]. Obviously if $G$ satisfies the strong Atiyah conjecture over $\mathbb{C}$, then $G$ satisfies the strong Atiyah conjecture over $K$ for all subfields $K$ of $\mathbb{C}$. The strong Atiyah conjecture over $\mathbb{C}$ is known for large classes of groups; for example [6, Theorem 1.5] tells us that it is true if $G$ has a normal free subgroup $F$ such that $G / F$ is an elementary amenable group. If $K$ is the algebraic closure of $\mathbb{Q}$ in $\mathbb{C}$, it is known for even larger classes of groups, for example [4, Theorem 1.4] for groups which are residually torsion-free elementary amenable. The following result is well known; see for example [13, Lemma 3].

Proposition 1.2. Let $G$ be a torsion-free group (i.e. $\operatorname{lcm}(G)=1$ ) and let $K$ be a subfield of $\mathbb{C}$. Then $G$ satisfies the strong Atiyah conjecture over $K$ if and only if $\mathcal{D}(K G)$ is a skew field.

The purpose of this paper is to generalize Proposition 1.2. We will denote the finite conjugate subgroup of the group $G$ by $\Delta(G)$, and the torsion subgroup of $\Delta(G)$ by $\Delta^{+}(G)$ (this is a subgroup, compare [12, Lemma 19.3]). We shall prove

Theorem 1.3. Let $G$ be a group with $d:=\operatorname{lcm}(G)<\infty$ and $\Delta^{+}(G)=1$, and let $K$ be a subfield of $\mathbb{C}$ that is closed under complex conjugation. Then $G$ satisfies the strong Atiyah conjecture over $K$ if and only if $\mathcal{D}(K G)$ is a $d \times d$ matrix ring over a skew field. 
It seems plausible that if $K$ is a subfield of $\mathbb{C}$ which is closed under complex conjugation and $G$ is a group with $\operatorname{lcm}(G)<\infty$ which satisfies the strong Atiyah conjecture over $K$, then $\mathcal{D}(K G)$ is a semisimple Artinian ring. However we cannot prove this, though we are able to prove a slightly weaker result, and to state this we require the following definition.

Definition 1.4. Let $R$ be a subring of the ring $S$. The extended division closure, $\mathcal{E}(R, S)$, of $R$ in $S$ is the smallest subring of $S$ containing $R$ with the properties

(a) If $x \in \mathcal{E}(R, S)$ and $x^{-1} \in S$, then $x \in \mathcal{E}(R, S)$.

(b) If $x \in \mathcal{E}(R, S)$ and $x S=e S$ where $e$ is a central idempotent of $S$, then $e \in \mathcal{E}(R, S)$.

Obviously $\mathcal{E}(R, S) \supseteq \mathcal{D}(R, S)$. Note that if $\left\{R_{i}\right\}$ is a collection of subrings of $S$ satisfying 1.4(a) and 1.4(b) above, then $\bigcap_{i} R_{i}$ is also a subring of $S$ satisfying 1.4(a) and 1.4(b), consequently $\mathcal{E}(R, S)$ is a well defined subring of $S$ containing $R$. Also if $G$ is a group and $K$ is a subfield of $\mathbb{C}$, then we write $\mathcal{E}(K G)$ for $\mathcal{E}(K G, \mathcal{U}(G))$. Observe that, if $G$ is torsion free and if the strong Atiyah conjecture holds for $G$ over $K$, then $\mathcal{D}(K G)$ is a division ring, hence $x \mathcal{D}(K G)=\mathcal{D}(K G)$ for every $0 \neq x \in \mathcal{D}(K G)$ and consequently $\mathcal{E}(K G)=\mathcal{D}(K G)$ in this case. We are tempted to conjecture that this is always the case. We hope to show in a later paper that this should follow from a suitable version of the Atiyah conjecture.

We shall prove

Theorem 1.5. Let $G$ be a group with $\operatorname{lcm}(G)<\infty$, and let $K$ be a subfield of $\mathbb{C}$ that is closed under complex conjugation. Suppose that $G$ satisfies the strong Atiyah conjecture over $K$. Then $\mathcal{E}(K G)$ is a semisimple Artinian ring.

Theorem 1.5 follows immediately from the more general Theorem 2.7 in Section 2. Thus in particular if $K$ is a subfield of $\mathbb{C}$ that is closed under complex conjugation and $G$ is a group with $\operatorname{lcm}(G)<\infty$ which satisfies the strong Atiyah conjecture over $K$, then $K G$ can be embedded in a semisimple Artinian ring. In fact we can remove the hypothesis that $K$ is closed under complex conjugation to obtain

Corollary 1.6. Let $G$ be a group with $\operatorname{lcm}(G)<\infty$ and let $K$ be a subfield of $\mathbb{C}$. Suppose that $G$ satisfies the strong Atiyah conjecture over $K$. Then $K G$ can be embedded in a semisimple Artinian ring. 
In Section 3 we will show, somewhat unrelated to the rest of the paper, that $K G$ can be embedded in a least subring of $\mathcal{U}(G)$ that is $*$-regular.

\section{Proofs}

Let $R$ be a subring of the ring $S$ and let $C=\{e \in S \mid e$ is a central idempotent of $S$ and $e S=r S$ for some $r \in R\}$. Then we define

$$
\mathcal{C}(R, S)=\sum_{e \in C} e R
$$

a subring of $S$. In the case $S=\mathcal{U}(G)$, we write $\mathcal{C}(R)$ for $\mathcal{C}(R, \mathcal{U}(G))$. For each ordinal $\alpha$, define $\mathcal{E}_{\alpha}(R, S)$ as follows:

- $\mathcal{E}_{0}(R, S)=R$

- $\mathcal{E}_{\alpha+1}(R, S)=\mathcal{D}\left(\mathcal{C}\left(\mathcal{E}_{\alpha}(R, S), S\right), S\right)$;

- $\mathcal{E}_{\alpha}(R, S)=\bigcup_{\beta<\alpha} \mathcal{E}_{\beta}(R, S)$ if $\alpha$ is a limit ordinal.

Then $\mathcal{E}(R, S)=\bigcup_{\alpha} \mathcal{E}_{\alpha}(R, S)$. Also in the case $R=K G$ where $G$ is a group and $K$ is a subfield of $\mathbb{C}$, we shall write $\mathcal{E}_{\alpha}(K G)$ for $\mathcal{E}_{\alpha}(K G, \mathcal{U}(G))$. If $A \subseteq \mathbb{R}$, then $\langle A\rangle$ will indicate the additive subgroup of $\mathbb{R}$ generated by $A$.

Lemma 2.1. Let $G$ be a group, let $R$ be a subring of $\mathcal{U}(G)$, let $n \in \mathbb{N}$, and let $x \in R$. Suppose that $x \mathcal{U}(G)=e \mathcal{U}(G)$ where $e$ is a central idempotent of $\mathcal{U}(G)$. Then $\left\langle\operatorname{dim}_{\mathcal{N}(G)} \beta \mathcal{U}(G)^{n} \mid \beta \in \mathrm{M}_{n}(R)\right\rangle=\left\langle\operatorname{dim}_{\mathcal{N}(G)} \alpha \mathcal{U}(G)^{n} \mid \alpha \in \mathrm{M}_{n}(R+e R)\right\rangle$.

Proof. Set $E=e I_{n}$, the diagonal matrix in $\mathrm{M}_{n}(R+e R)$ that has $e$ 's on the main diagonal and zeros elsewhere. Then $E$ is a central idempotent in $\mathrm{M}_{n}(\mathcal{U}(G))$. Obviously

$$
\left\langle\operatorname{dim}_{\mathcal{N}(G)} \beta \mathcal{U}(G)^{n} \mid \beta \in \mathrm{M}_{n}(R)\right\rangle \subseteq\left\langle\operatorname{dim}_{\mathcal{N}(G)} \alpha \mathcal{U}(G)^{n} \mid \alpha \in \mathrm{M}_{n}(R+e R)\right\rangle,
$$

so we need to prove the reverse inclusion. Let $\alpha \in \mathrm{M}_{n}(R+e R)$ and write $\alpha=\beta+E \gamma$ where $\beta, \gamma \in \mathrm{M}_{n}(R)$. Then we have

$$
\operatorname{dim}_{\mathcal{N}(G)} \alpha \mathcal{U}(G)^{n}=\operatorname{dim}_{\mathcal{N}(G)}(\beta+\gamma) E \mathcal{U}(G)^{n}+\operatorname{dim}_{\mathcal{N}(G)} \beta(1-E) \mathcal{U}(G)^{n} .
$$

Since $\operatorname{dim}_{\mathcal{N}(G)} \beta(1-E) \mathcal{U}(G)^{n}=\operatorname{dim}_{\mathcal{N}(G)} \beta \mathcal{U}(G)^{n}-\operatorname{dim}_{\mathcal{N}(G)} \beta E \mathcal{U}(G)^{n}$, it suffices to prove that

$$
\operatorname{dim}_{\mathcal{N}(G)} E \beta \mathcal{U}(G)^{n} \in\left\langle\operatorname{dim}_{\mathcal{N}(G)} \delta \mathcal{U}(G)^{n} \mid \delta \in \mathrm{M}_{n}(R)\right\rangle
$$

for all $\beta \in \mathrm{M}_{n}(R)$. But $E \beta \mathcal{U}(G)^{n}=\beta\left(x I_{n}\right) \mathcal{U}(G)^{n}$ and the result follows. 
Lemma 2.1 immediately gives the following corollary.

Corollary 2.2. Let $G$ be a group, let $R$ be a subring of $\mathcal{U}(G)$, and let $n \in \mathbb{N}$. Then $\left\langle\operatorname{dim}_{\mathcal{N}(G)} \alpha \mathcal{U}(G)^{n} \mid \alpha \in \mathrm{M}_{n}(R)\right\rangle=\left\langle\operatorname{dim}_{\mathcal{N}(G)} \alpha \mathcal{U}(G)^{n} \mid \alpha \in \mathrm{M}_{n}(\mathcal{C}(R))\right\rangle$.

Proof. Let $e_{1}, \ldots, e_{m}$ be central idempotents of $\mathcal{U}(G)$ such that for each $i$, there exists $\alpha_{i} \in R$ with $e_{i} \mathcal{U}(G)=\alpha_{i} \mathcal{U}(G)$. Then by induction on $m$, Lemma 2.1 tells us that the result is true if $\alpha \in \mathrm{M}_{n}\left(R+e_{1} R+\cdots+e_{m} R\right)$. Since $\mathrm{M}_{n}(\mathcal{C}(R))$ is the union of $\mathrm{M}_{n}\left(R+e_{1} R+\cdots+e_{m} R\right)$, the result is proven.

Lemma 2.3. Let $R$ be a subring of the ring $S$, let $n \in \mathbb{N}$, and let $A \in \mathrm{M}_{n}(\mathcal{D}(R, S))$. Then there exist $0 \leq m \in \mathbb{Z}$ and $X, Y \in \mathrm{GL}_{m+n}(S)$ such that $X \operatorname{diag}\left(A, I_{m}\right) Y \in$ $\mathrm{M}_{m+n}(R)$.

Proof. This follows from [3, Proposition 7.1.3 and Exercise 7.1.4] and [7, Proposition 3.4].

Lemma 2.4. Let $G$ be a group and let $K$ be a subfield of $\mathbb{C}$. Then

$$
\begin{aligned}
\left\langle\operatorname{dim}_{\mathcal{N}(G)} x \mathcal{U}(G)^{n} \mid x \in \mathrm{M}_{n}(K G), n \in \mathbb{N}\right\rangle & \\
& =\left\langle\operatorname{dim}_{\mathcal{N}(G)} x \mathcal{U}(G)^{n} \mid x \in \mathrm{M}_{n}(\mathcal{E}(K G)), n \in \mathbb{N}\right\rangle
\end{aligned}
$$

Proof. Obviously

$$
\begin{aligned}
\left\langle\operatorname{dim}_{\mathcal{N}(G)} x \mathcal{U}(G)^{n} \mid x \in \mathrm{M}_{n}(K G), n \in \mathbb{N}\right\rangle & \\
& \subseteq\left\langle\operatorname{dim}_{\mathcal{N}(G)} x \mathcal{U}(G)^{n} \mid x \in \mathrm{M}_{n}(\mathcal{E}(K G)), n \in \mathbb{N}\right\rangle
\end{aligned}
$$

We shall prove the reverse inclusion by transfinite induction. So let $n \in \mathbb{N}$ and $x \in \mathrm{M}_{n}(\mathcal{E}(K G))$. Then we may choose the least ordinal $\alpha$ such that $x \in$ $\mathrm{M}_{n}\left(\mathcal{E}_{\alpha}(K G)\right)$. Clearly $\alpha$ is not a limit ordinal, and the result is true if $\alpha=0$, so we may write $\alpha=\beta+1$ for some ordinal $\beta$ and assume that the result is true for all $y \in \mathrm{M}_{n}\left(\mathcal{E}_{\beta}(K G)\right)$. By Corollary 2.2 the result is true for all $y \in \mathrm{M}_{n}\left(\mathcal{C}\left(\mathcal{E}_{\beta}(K G)\right)\right)$ and now the result follows from Lemma 2.3.

The following result from [8] will be crucial for our work here. Because of this, and because we use a slightly different formulation, we state it here. 
Lemma 2.5. [8, Lemma 2] Let $G$ be a group, let $n \in \mathbb{N}$, and let $\alpha_{1}, \ldots, \alpha_{n} \in$ $\mathcal{U}(G)$. Then $\left(\sum_{j=1}^{n} \alpha_{j} \alpha_{j}^{*}\right) \mathcal{U}(G) \supseteq \alpha_{1} \mathcal{U}(G)$. It then also follows that

$$
\left(\sum_{j=1}^{n} \alpha_{j} \alpha_{j}^{*}\right) \mathcal{U}(G)=\sum_{j=1}^{n} \alpha_{j} \alpha_{j}^{*} \mathcal{U}(G)=\sum_{j=1}^{n} \alpha_{j} \mathcal{U}(G) .
$$

Proof. The case $n=2$ in the first statement is [8, Lemma 2]; the proof there can easily be modified to give the case for general $n$. Alternatively we can argue as follows: given $\alpha, \beta \in \mathcal{U}(G)$, there exists $\gamma \in \mathcal{U}(G)$ such that $\alpha \alpha^{*}+\beta \beta^{*}=\gamma \gamma^{*}$, by [2, Proposition 3 of Section 53 on p. 239 and Remark 1 of Section 55 on p. 249], because $\mathcal{N}(G)$ is an $A W^{*}$-algebra. Also it is obvious that $\alpha \mathcal{U}(G) \supseteq \alpha \alpha^{*} \mathcal{U}(G)$ and hence $\alpha \mathcal{U}(G)=\alpha \alpha^{*} \mathcal{U}(G)$. Using these facts and induction on $n$, we obtain the first statement of the lemma. Replacing 1 with $j$ in the right hand side, we see that

$$
\alpha_{j} \mathcal{U}(G)=\alpha_{j} \alpha_{j}^{*} \mathcal{U}(G) \subseteq\left(\sum_{j=1}^{n} \alpha_{j} \alpha_{j}^{*}\right) \mathcal{U}(G),
$$

and equality (2.6) follows.

Theorem 2.7. Let $G$ be a group and let $K$ be a subfield of $\mathbb{C}$ which is closed under complex conjugation. Suppose there is an $\ell \in \mathbb{N}$ such that $\ell \operatorname{dim}_{\mathcal{N}(G)} \alpha \mathcal{U}(G)^{n} \in \mathbb{Z}$ for all $\alpha \in \mathrm{M}_{n}(K G)$ and for all $n \in \mathbb{N}$. Then $\mathcal{E}(K G)$ is a semisimple Artinian ring.

Proof. First observe that Lemma 2.4 tells us that

$$
\ell \operatorname{dim}_{\mathcal{N}(G)} \alpha \mathcal{U}(G) \in \mathbb{Z} \text { for all } \alpha \in \mathcal{E}(K G) .
$$

Next note that the hypothesis tells us that $\mathcal{E}(K G)$ has at most $\ell$ primitive central idempotents. Indeed if $e_{1}, \ldots, e_{\ell+1}$ are (nonzero distinct) primitive central idempotents, then $e_{i} e_{j}=0$ for $i \neq j$ and we see that the sum $\bigoplus_{i=1}^{\ell+1} e_{i} \mathcal{U}(G)$ is direct. But

$$
\operatorname{dim}_{\mathcal{N}(G)} \bigoplus_{i=1}^{\ell+1} e_{i} \mathcal{U}(G)=\sum_{i=1}^{\ell+1} \operatorname{dim}_{\mathcal{N}(G)} e_{i} \mathcal{U}(G) \geq(\ell+1) / \ell>1
$$

by (2.8), and we have a contradiction. Thus $\mathcal{E}(K G)$ has $n$ primitive central idempotents $e_{1}, \ldots, e_{n}$ for some $n \in \mathbb{N}, n \leq \ell$. For each $i, 1 \leq i \leq n$, choose $0 \neq \alpha_{i} \in e_{i} \mathcal{E}(K G)$ such that $\operatorname{dim}_{\mathcal{N}(G)} \alpha_{i} \mathcal{U}(G)$ is minimal. 
Fix $m \in\{1,2, \ldots, n\}$. Since $\ell \operatorname{dim}_{\mathcal{N}(G)} \alpha \mathcal{U}(G) \in \mathbb{Z}$ for all $\alpha \in \mathcal{E}(K G)$ by (2.8), we may choose $g_{1}, \ldots, g_{r} \in G$ with $\operatorname{dim}_{\mathcal{N}(G)}\left(\sum_{i=1}^{r} g_{i} \alpha_{m} \alpha_{m}^{*} g_{i}^{-1}\right) \mathcal{U}(G)$ maximal. Note that if $g_{r+1} \in G$, then

$$
\left(\sum_{i=1}^{r+1} g_{i} \alpha_{m} \alpha_{m}^{*} g_{i}^{-1}\right) \mathcal{U}(G) \supseteq \sum_{i=1}^{r} g_{i} \alpha_{m} \mathcal{U}(G) \supseteq\left(\sum_{i=1}^{r} g_{i} \alpha_{m} \alpha_{m}^{*} g_{i}^{-1}\right) \mathcal{U}(G)
$$

by Lemma 2.5 , hence

$$
\operatorname{dim}_{\mathcal{N}(G)}\left(\sum_{i=1}^{r+1} g_{i} \alpha_{m} \alpha_{m}^{*} g_{i}^{-1}\right) \mathcal{U}(G) \geq \operatorname{dim}_{\mathcal{N}(G)}\left(\sum_{i=1}^{r} g_{i} \alpha_{m} \alpha_{m}^{*} g_{i}^{-1}\right) \mathcal{U}(G)
$$

and by maximality of $\operatorname{dim}_{\mathcal{N}(G)}\left(\sum_{i=1}^{r} g_{i} \alpha_{m} \alpha_{m}^{*} g_{i}^{-1}\right) \mathcal{U}(G)$, we see that

$$
\operatorname{dim}_{\mathcal{N}(G)}\left(\sum_{i=1}^{r+1} g_{i} \alpha_{m} \alpha_{m}^{*} g_{i}^{-1}\right) \mathcal{U}(G)=\operatorname{dim}_{\mathcal{N}(G)}\left(\sum_{i=1}^{r} g_{i} \alpha_{m} \alpha_{m}^{*} g_{i}^{-1}\right) \mathcal{U}(G)
$$

It follows that

$$
\left(\sum_{i=1}^{r+1} g_{i} \alpha_{m} \alpha_{m}^{*} g_{i}^{-1}\right) \mathcal{U}(G)=\left(\sum_{i=1}^{r} g_{i} \alpha_{m} \alpha_{m}^{*} g_{i}^{-1}\right) \mathcal{U}(G)
$$

and we deduce from Lemma 2.5 that $g \alpha_{m} \mathcal{U}(G) \subseteq\left(\sum_{i=1}^{r} g_{i} \alpha_{m} \alpha_{m}^{*} g_{i}^{-1}\right) \mathcal{U}(G)$ for all $g \in G$. Let $f \in \mathcal{U}(G)$ be the unique projection such that

$$
f \mathcal{U}(G)=\sum_{i=1}^{r} g_{i} \alpha_{m} \alpha_{m}^{*} g_{i}^{-1} \mathcal{U}(G)
$$

Then $g f \mathcal{U}(G)=\sum g g_{i} \alpha_{m} \alpha_{m}^{*} g_{i}^{-1} \mathcal{U}(G) \subseteq \sum g g_{i} \alpha_{m} \mathcal{U}(G) \subseteq f \mathcal{U}(G)$ for all $g \in G$, thus $g f \mathcal{U}(G)=f \mathcal{U}(G)$ and we deduce that $g f g^{-1} \mathcal{U}(G)=f \mathcal{U}(G)$ for all $g \in G$. Also $g g^{-1}$ is also a projection, thus $g f g^{-1}=f$ for all $g \in G$ and we conclude that $f$ is a central projection in $\mathcal{E}(K G)$. Since $f \neq 0, f \mathcal{U}(G) \subseteq e_{m} \mathcal{U}(G)$ and $e_{m}$ is primitive, we conclude that $f=e_{m}$ and consequently $\sum_{i=1}^{r} g_{i} \alpha_{m} \mathcal{U}(G)=e_{m} \mathcal{U}(G)$. By omitting some of the terms in this sum if necessary, we may assume that

$$
\sum_{1 \leq i \leq r, i \neq s} g_{i} \alpha_{m} \mathcal{U}(G) \neq e_{m} \mathcal{U}(G)
$$

for all $s$ such that $1 \leq s \leq r$. We make the following observation:

$$
\text { If } 0 \neq x \in g_{s} \alpha_{m} \mathcal{E}(K G) \text {, then } x \mathcal{U}(G)=g_{s} \alpha_{m} \mathcal{U}(G) \text {, }
$$

where $1 \leq s \leq r$. This is because $0 \neq x \mathcal{U}(G) \subseteq g_{s} \alpha_{m} \mathcal{U}(G)$ and by minimality of $\operatorname{dim}_{\mathcal{N}(G)} \alpha_{m} \mathcal{U}(G)$, we see that $\operatorname{dim}_{\mathcal{N}(G)} x \mathcal{U}(G)=\operatorname{dim}_{\mathcal{N}(G)} g_{s} \alpha_{m} \mathcal{U}(G)$ and consequently $x \mathcal{U}(G)=g_{s} \alpha_{m} \mathcal{U}(G)$. 
We claim that $e_{m} \mathcal{E}(K G)=\bigoplus_{i=1}^{r} g_{i} \alpha_{m} \mathcal{E}(K G)$. Set $\sigma=\left(\sum_{i=1}^{r} g_{i} \alpha_{m} \alpha_{m}^{*} g_{i}^{-1}\right)$. Since $\sigma \mathcal{U}(G)=e_{m} \mathcal{U}(G)$, we see that

$\left(\sigma+\left(1-e_{m}\right)\right) \mathcal{U}(G) \supseteq \sigma \mathcal{U}(G)+\left(1-e_{m}\right) \mathcal{U}(G)=e_{m} \mathcal{U}(G)+\left(1-e_{m}\right) \mathcal{U}(G)=\mathcal{U}(G)$.

Therefore, $\sigma+1-e_{m}$ is invertible in $\mathcal{U}(G)$ and hence $\sigma+1-e_{m}$ is invertible in $\mathcal{E}(K G)$. Thus

$$
e_{m} \sigma \mathcal{E}(K G)=e_{m}\left(\sigma+1-e_{m}\right) \mathcal{E}(K G)=e_{m} \mathcal{E}(K G) .
$$

Moreover, $\sigma \mathcal{E}(K G) \subseteq e_{m} \mathcal{E}(K G)$ and therefore $e_{m} \sigma \mathcal{E}(K G)=\sigma \mathcal{E}(K G)$, hence

$$
e_{m} \mathcal{E}(K G)=\sigma \mathcal{E}(K G)=\sum_{i=1}^{r} g_{i} \alpha_{m} \mathcal{E}(K G) .
$$

If this sum is not direct, then for some $s$ with $1 \leq s \leq r$, we have $g_{s} \alpha_{m} \mathcal{E}(K G) \cap$ $\sum_{i \neq s} g_{i} \alpha_{m} \mathcal{E}(K G) \neq 0$, and without loss of generality we may assume that $s=1$.

So let $0 \neq x \in g_{1} \alpha_{m} \mathcal{E}(K G) \cap \sum_{i=2}^{r} g_{i} \alpha_{m} \mathcal{E}(K G)$. Then $0 \neq x \mathcal{U}(G) \subseteq g_{1} \alpha_{m} \mathcal{U}(G)$ and (2.10) shows that $x \mathcal{U}(G)=g_{1} \alpha_{m} \mathcal{U}(G)$. It follows that $g_{1} \alpha_{m} \mathcal{U}(G) \subseteq \sum_{i=2}^{r} g_{i} \alpha_{m} \mathcal{U}(G)$, consequently

$$
\sum_{i=2}^{r} g_{i} \alpha_{m} \mathcal{U}(G)=e_{m} \mathcal{U}(G),
$$

which contradicts (2.9) and our claim is established.

Now we show that $g_{1} \alpha_{m} \mathcal{E}(K G)$ is an irreducible $\mathcal{E}(K G)$-module. Suppose $0 \neq x \in g_{1} \alpha_{m} \mathcal{E}(K G)$. Then $x \mathcal{U}(G)=g_{1} \alpha_{m} \mathcal{U}(G)$ by (2.10) and using Lemma 2.5, we see as before that $x x^{*}+\sum_{i=2}^{r} g_{i} \alpha_{i} \alpha_{i}^{*} g_{i}^{-1}+1-e_{m}$ is a unit in $\mathcal{U}(G)$ and hence is also a unit in $\mathcal{E}(K G)$. This proves that $x \mathcal{E}(K G)=g_{1} \alpha_{m} \mathcal{E}(K G)$ and we deduce that $\mathcal{E}(K G)$ is a finite direct sum of irreducible $\mathcal{E}(K G)$-modules. It follows that $\mathcal{E}(K G)$ is a semisimple Artinian ring.

Proof of Corollary 1.6. Set $k=K \cap \mathbb{R}$, the maximal real subfield of $K$. If $k=K$, then the result is obvious from Theorem 1.5, so we may assume that $k \neq K$. In this case the degree of $K$ over $k$ will be 2 and we may write $K=k(\alpha)$ where $\alpha^{2} \in k$. Clearly $G$ satisfies the strong Atiyah over $k$, so we may embed $k G$ into a semisimple Artinian ring $A$ by Theorem 1.5. Now we may embed $K G$ into $\mathrm{M}_{2}(k G)$ in the standard way, which we now describe. Let $F$ denote the free right $k G$-module with basis $\{e, f\}$. Then if $w \in K G$, we may write $w=u+\alpha v$ where $u, v \in k G$, and we define a right $k G$-module map of $F$ by $w e=e u+f v$ and 
$w f=e \alpha^{2} v+f u$. It is easily checked that $K G$ acts on the left of $F$ by right $k G$-modules maps, and it follows that we have embedded $K G$ into $\mathrm{M}_{2}(k G)$. We deduce that $K G$ embeds into $\mathrm{M}_{2}(A)$. Since $A$ is semisimple Artinian, this matrix ring is also semisimple Artinian [11, Proposition 3.5.10 on p. 85], and the result follows.

Proposition 2.11. Let $G$ be a group with $\Delta(G)$ finite and let $K$ be a subfield of $\mathbb{C}$ which is closed under complex conjugation and contains all $|\Delta(G)|$-th roots of unity, e.g. $K=\mathbb{C}$ or $K$ is the algebraic closure of $\mathbb{Q}$ in $\mathbb{C}$. Then $\mathcal{E}(K G)=$ $\mathcal{D}(K G)$.

Proof. If $e$ is a central idempotent in $\mathcal{U}(G)$, then $e \in \mathcal{N}(\Delta(G))$, in particular $e \in \mathbb{C} G$, and by our assumption on $K$ even $e \in K G$. The result follows.

The following result is well known, but we include a proof.

Lemma 2.12. Let $G$ be a group, let e be a projection in $\mathcal{N}(G)$, and let $\alpha \in \mathcal{N}(G)$. Then $\operatorname{tr}\left(e \alpha \alpha^{*} e\right) \leq \operatorname{tr}\left(\alpha \alpha^{*}\right)$.

Proof. Since $\operatorname{tr}(x y)=\operatorname{tr}(y x)$ for all $x, y \in \mathcal{N}(G)$, we see that $\operatorname{tr}\left(e \alpha \alpha^{*}(1-e)\right)=$ $\operatorname{tr}\left((1-e) \alpha \alpha^{*} e\right)=0$. Therefore $\operatorname{tr}\left(\alpha \alpha^{*}\right)=\operatorname{tr}\left(e \alpha \alpha^{*} e\right)+\operatorname{tr}\left((1-e) \alpha \alpha^{*}(1-e)\right)$. Since $\operatorname{tr}\left((1-e) \alpha \alpha^{*}(1-e)\right) \geq 0$, the result follows.

Lemma 2.13. Let $G$ be a group, and let $\left(\alpha_{n}\right)$ be a sequence in $\mathcal{N}(G)$ converging strongly to $\alpha$. Suppose that $\operatorname{ker} \alpha=0$. Then $\operatorname{dim}_{\mathcal{N}(G)}\left(\operatorname{ker} \alpha_{n}\right)$ converges to 0 .

Proof. By the principle of uniform boundedness, $\left\|\alpha_{n}\right\|$ is bounded. Also by multiplying everything by a unitary operator if necessary, we may assume that $\alpha$ is positive. Then $\alpha_{n}-\alpha$ converges strongly to 0 and $\left(\alpha_{n}-\alpha\right)^{*}$ is bounded, hence $\left(\alpha_{n}-\alpha\right)^{*}\left(\alpha_{n}-\alpha\right)$ converges strongly to 0 and in particular $\lim _{n \rightarrow \infty} \operatorname{tr}\left(\left(\alpha_{n}-\right.\right.$ $\left.\alpha)^{*}\left(\alpha_{n}-\alpha\right)\right)=0$. Let $e_{n} \in \mathcal{N}(G)$ denote the projection of $\ell^{2}(G)$ onto $\operatorname{ker} \alpha_{n}$. Then $e_{n} \alpha_{n}^{*}=\alpha_{n} e_{n}=0$ and using Lemma 2.12, we obtain

$$
\begin{aligned}
\operatorname{tr}\left(\left(\alpha_{n}-\alpha\right)^{*}\left(\alpha_{n}-\alpha\right)\right) & \geq \operatorname{tr}\left(e_{n}\left(\alpha_{n}-\alpha\right)^{*}\left(\alpha_{n}-\alpha\right) e_{n}\right) \\
& =\operatorname{tr}\left(e_{n} \alpha^{*} \alpha e_{n}\right) \geq 0 .
\end{aligned}
$$

Thus $\lim _{n \rightarrow \infty} \operatorname{tr}\left(e_{n} \alpha^{*} \alpha e_{n}\right)=0$. Suppose by way of contradiction that $\lim _{n \rightarrow \infty} \operatorname{dim}_{\mathcal{N}(G)}\left(\operatorname{ker} \alpha_{n}\right) \neq 0$. Then by taking a subsequence if necessary, we may assume that $\operatorname{dim}_{\mathcal{N}(G)}\left(\operatorname{ker} \alpha_{n}\right)>\epsilon$ for some $\epsilon>0$, for all $n \in \mathbb{N}$. By 
considering the spectral family associated to $\alpha^{*} \alpha$ [10, Definition 1.68], there is a closed $\alpha^{*} \alpha$-invariant $\mathcal{N}(G)$-submodule $X$ of $\ell^{2}(G)$ and a $\delta>0$ such that $\operatorname{dim}_{\mathcal{N}(G)}(X)>1-\epsilon / 2$ and $\alpha^{*} \alpha>\delta$ on $X$. Because $\operatorname{dim}_{\mathcal{N}(G)}(X)>1-\epsilon / 2$ and $\operatorname{dim}_{\mathcal{N}(G)}\left(\operatorname{ker} \alpha_{n}\right)>\epsilon$, we find that $\operatorname{dim}_{\mathcal{N}(G)}\left(X \cap \operatorname{ker} \alpha_{n}\right)>\epsilon / 2$ (use [10, Theorem $6.7])$. Let $f_{n}$ denote the projection of $\ell^{2}(G)$ onto $X \cap \operatorname{ker} \alpha_{n}$, so $\operatorname{tr} f_{n}>\epsilon / 2$. Since $\alpha^{*} \alpha>\delta$ on $X \cap \operatorname{ker} \alpha_{n}, f_{n} \alpha^{*} \alpha f_{n} \geq \delta f_{n}$, and because of positivity of $\operatorname{tr}$ we see that $\operatorname{tr}\left(f_{n} \alpha^{*} \alpha f_{n}\right) \geq \operatorname{tr}\left(\delta f_{n}\right)>\delta \epsilon / 2$. Therefore $\operatorname{tr}\left(e_{n} \alpha^{*} \alpha e_{n}\right)>\epsilon \delta / 2$ by Lemma 2.12, which shows that $\operatorname{tr}\left(e_{n} \alpha^{*} \alpha e_{n}\right)$ does not converge to 0 , and the result follows.

Proposition 2.14. Let $G$ be a group with $\Delta^{+}(G)=1$ and let $K$ be a subfield of $\mathbb{C}$ that is closed under complex conjugation. Assume that $\operatorname{lcm}(G)=d \in \mathbb{N}$ and that $G$ satisfies the strong Atiyah conjecture over $K$. Then $\mathcal{D}(K G)$ is a $d \times d$ matrix ring over a skew field.

Proof. Let $p$ be a prime, let $q$ be the largest power of $p$ that divides $d$, and let $H \leq G$ with $|H|=q$ (so $H$ is a "Sylow" $p$-subgroup of $G$ ). Set $e=\frac{1}{q} \sum_{h \in H} h$, a projection in $\mathbb{Q} H$. We shall use the center valued von Neumann dimension $\operatorname{dim}^{u}$, as defined in [10, Definition 9.12]. Since $\Delta^{+}(G)=1$, we see that $\operatorname{dim}^{u}(e \mathcal{U}(G))=$ $1 / q$ and $\operatorname{dim}^{u}((1-e) \mathcal{U}(G))=(q-1) / q$. Therefore by [10, Theorem 9.13(1)],

$$
(1-e) \mathcal{U}(G) \cong e \mathcal{U}(G)^{q-1}
$$

and we deduce that there exist orthogonal projections $e=e_{1}, e_{2}, \ldots, e_{q} \in \mathcal{U}(G)$ (so $e_{i} e_{j}=0$ for $i \neq j$ ) such that $\sum_{i=1}^{q} e_{i}=1$ and $e_{i} \mathcal{U}(G) \cong e \mathcal{U}(G)$ for all $i$. By [2, Exercise 13.15A, p. 76], there exist similarities (that is self adjoint unitaries) $u_{i} \in \mathcal{U}(G)$ with $u_{1}=1$ such that $e_{i}=u_{i} e u_{i}$. There is a countable subgroup $F$ of $G$ such that $u_{i} \in \mathcal{N}(F)$ for all $i$. By the Kaplansky density theorem [1, Corollary, p. 8] for each $i(1 \leq i \leq q)$ there exists a sequence $u_{i j} \in K F$ such that $u_{i j} \rightarrow u_{i}$ as $j \rightarrow \infty$ in the strong operator topology in $\mathcal{N}(F)$ with $u_{1 j}=1$ for all $j$. Set $v_{j}=\sum_{i=1}^{q} u_{i j} e u_{i j}$. Then $v_{j} \rightarrow \sum_{i=1}^{q} e_{i}=1$ strongly, hence for $1 \leq i \leq q$,

$$
\lim _{j \rightarrow \infty} \operatorname{dim}_{\mathcal{N}(F)}\left(v_{j} \mathcal{U}(F)\right)=\lim _{j \rightarrow \infty} \operatorname{dim}_{\mathcal{N}(F)}\left(u_{i j} \mathcal{U}(F)\right)=1
$$

by Lemma 2.13. Now $\operatorname{dim}_{\mathcal{N}(F)}(x \mathcal{U}(F))=\operatorname{dim}_{\mathcal{N}(G)}(x \mathcal{U}(G))$ for all $x \in \mathcal{U}(F)$, consequently

$$
\lim _{j \rightarrow \infty} \operatorname{dim}_{\mathcal{N}(G)} v_{j} \mathcal{U}(G)=\lim _{j \rightarrow \infty} \operatorname{dim}_{\mathcal{N}(G)}\left(u_{i j} \mathcal{U}(G)\right)=1 \quad \text { for } 1 \leq i \leq q,
$$

and since by assumption $G$ satisfies the strong Atiyah conjecture over $K$, there exists $n \in \mathbb{N}$ such that $\operatorname{dim}_{\mathcal{N}(G)} v_{j} \mathcal{U}(G)=\operatorname{dim}_{\mathcal{N}(G)}\left(u_{i j} \mathcal{U}(G)\right)=1$ for $1 \leq i \leq q$ 
for all $j \geq n$, in particular $\operatorname{dim}_{\mathcal{N}(G)}\left(v_{n} \mathcal{U}(G)\right)=\operatorname{dim}_{\mathcal{N}(G)}\left(u_{i n} \mathcal{U}(G)\right)=1$ and we conclude that $v_{n}$ and $u_{i n}(1 \leq i \leq q)$ are units in $\mathcal{U}(G)$. Therefore $v_{n}$ and $u_{i n}$ $(1 \leq i \leq q)$ are units in $\mathcal{D}(K G)$ and we deduce that $\sum_{i=1}^{q} u_{\text {in }} e \mathcal{D}(K G)=\mathcal{D}(K G)$, because

$$
D(K G)=v_{n} D(K G)=\sum_{i=1}^{q}\left(u_{i n} e u_{i n}\right) D(K G) \subseteq \sum_{i=1}^{q} u_{i n} e D(K G) \subseteq D(K G) .
$$

Since $\operatorname{dim}_{\mathcal{N}(G)} e \mathcal{U}(G)=1 / q$, we see that $\bigoplus_{i=1}^{q} u_{\text {in }} e \mathcal{U}(G)=\mathcal{U}(G)$, a direct sum, and we deduce that

$$
\bigoplus_{i=1}^{q} u_{i n} e \mathcal{D}(K G)=\mathcal{D}(K G)
$$

also a direct sum.

Now suppose that $\varepsilon$ is a central idempotent in $\mathcal{C}(\mathcal{D}(K G))$. We want to prove that $\varepsilon=0$ or 1 , so assume otherwise. Now $\varepsilon u_{i n} e \mathcal{U}(G) \cong \varepsilon e \mathcal{U}(G)$ for all $i$, which implies that $\operatorname{dim}_{\mathcal{N}(G)}(\varepsilon \mathcal{U}(G))=q \operatorname{dim}_{\mathcal{N}(G)}(\varepsilon \mathcal{U}(G))$. Moreover, because of the Atiyah conjecture, $d \operatorname{dim}_{\mathcal{N}(G)}(\varepsilon e \mathcal{U}(G)) \in \mathbb{Z}$. These two observations together imply that $d \operatorname{dim}_{\mathcal{N}(G)}(\varepsilon \mathcal{U}(G)) \in q \mathbb{Z}$. Since this is true for all primes $p$, it follows that $\operatorname{dim}_{\mathcal{N}(G)} \mathcal{E}(G) \in \mathbb{Z}$, so 0 and 1 are the only central idempotents of $\mathcal{C}(\mathcal{D}(K G))$.

Summing up, we have shown that $\mathcal{C}(\mathcal{D}(K G))$ contains no nontrivial central idempotents. Using Theorem 2.7, we see that $\mathcal{D}(K G)$ is a semisimple Artinian ring with no nontrivial central idempotents. Thus $\mathcal{D}(K G)$ is an $l \times l$ matrix ring over a division ring for some $l \in \mathbb{N}$. In particular, $\mathcal{D}(K G)$ is the direct sum of $l$ mutually isomorphic $\mathcal{D}(K G)$-submodules, so if $f$ is a primitive idempotent in $\mathcal{D}(K G)$, we see that $\operatorname{dim}_{\mathcal{N}(G)}(f \mathcal{U}(G))=1 / l$. Furthermore Lemma 2.3 (or Lemma 2.4 ) show that $l \mid d$. On the other hand (2.15) shows that $q \mid l$, for all primes $p$, so $d \mid l$ and the result follows.

Proof of Theorem 1.3. If $G$ satisfies the strong Atiyah conjecture over $K$, then $\mathcal{D}(K G)$ is a $d \times d$ matrix ring over a skew field by Proposition 2.14. Conversely suppose $\mathcal{D}(K G)$ is a $d \times d$ matrix ring over a skew field $F$. We need to show that if $M$ is a finitely presented $K G$-module, then $\operatorname{lcm}(G) \operatorname{dim}_{\mathcal{N}(G)} M \otimes_{K G} \mathcal{U}(G) \in \mathbb{Z}$. However

$$
M \otimes_{K G} \mathcal{U}(G) \cong M \otimes_{K G} \mathrm{M}_{d}(F) \otimes_{\mathrm{M}_{d}(F)} \otimes \mathcal{U}(G),
$$

consequently $\left(M \otimes_{K G} \mathcal{U}(G)\right)^{d}$ is a finitely generated free $\mathcal{U}(G)$-module and we conclude that $\operatorname{lcm}(G) \operatorname{dim}_{\mathcal{N}(G)} M \otimes_{K G} \mathcal{U}(G) \in \mathbb{Z}$ as required. 


\section{EMBEDDINGS IN $*$-REGULAR RINGS}

There are other closures of group rings $K G$ in $\mathcal{U}(G)$ which may be useful, especially when $\operatorname{lcm}(G)=\infty$. In general the intersection of regular subrings of a von Neumann regular ring is not regular [5, Example 1.10], however we do have the following result.

Proposition 3.1. Let $G$ be a group and let $\left\{R_{i} \mid i \in \mathcal{I}\right\}$ be a collection of *-regular subrings of $\mathcal{U}(G)$. Then $\bigcap_{i \in \mathcal{I}} R_{i}$ is also a $*$-regular subring of $\mathcal{U}(G)$.

Proof. Set $S=\bigcap_{i \in \mathcal{I}} R_{i}$. Obviously $S$ is a $*$-subring of $\mathcal{U}(G)$; we need to show that $S$ is *-regular, that is given $s \in S$, there is a projection $e \in S$ such that $s S=e S$. We note that $\mathcal{D}\left(R_{i}, \mathcal{U}(G)\right)=R_{i}$ for all $i$. Indeed if $x \in R_{i}$ and $x$ is invertible in $\mathcal{U}(G)$, then $x R_{i}=e R_{i}$ where $e$ is a projection in $R_{i}$, consequently $x \mathcal{U}(G)=e \mathcal{U}(G)$ and since $x$ is invertible in $\mathcal{U}(G)$, we must have $e=1$ and we deduce that $x R_{i}=R_{i}$. Similarly $R_{i} x=R_{i}$ and thus $x$ is invertible in $R_{i}$, so $\mathcal{D}\left(R_{i}, \mathcal{U}(G)\right)=R_{i}$ as asserted. Since $R_{i}$ is $*$-regular, for each $i \in \mathcal{I}$, there is a projection $e_{i} \in R_{i}$ such that $e_{i} R_{i}=s R_{i}$. We now have $e_{i} \mathcal{U}(G)=e_{j} \mathcal{U}(G)$ for all $i, j$ and we deduce that $e_{i}=e_{j}$ for all $i, j \in \mathcal{I}$, so there exists $f \in S$ such that $f=e_{i}$ for all $i$. Since $f \mathcal{U}(G)=s \mathcal{U}(G)$, we see that $f s=s$, so $s \in f S$ and hence $s S \subseteq f S$. Thus the result will be proven if we can show that $s s^{*} S \supseteq f S$. By Lemma 2.5,

$$
\left(s s^{*}+(1-f)\right) \mathcal{U}(G) \supseteq(1-f) \mathcal{U}(G)+s \mathcal{U}(G)=(1-f) \mathcal{U}(G)+f \mathcal{U}(G)=\mathcal{U}(G)
$$

and we see that $s s^{*}+1-f$ is a unit in $\mathcal{U}(G)$. Let $t \in \mathcal{U}(G)$ be the inverse of $s s^{*}+1-f$, so

$$
\left(s s^{*}+1-f\right) t=1 \text {. }
$$

Since $\mathcal{D}\left(R_{i}, \mathcal{U}(G)\right)=R_{i}$ for all $i$, we deduce that $t \in R_{i}$ for all $i$ and hence $t \in S$. Moreover $f s=s$ and $f(1-f)=0$, so if we multiply (3.2) on the left by $f$, we obtain $s s^{*} t=f$ and the result is proven.

Thus if $K$ is a subfield of $\mathbb{C}$ that is closed under complex conjugation and $G$ is any group, then there is a least subring of $\mathcal{U}(G)$ containing $K G$ that is $*$-regular. 


\section{REFERENCES}

[1] William Arveson. An invitation to $C^{*}$-algebras. Springer-Verlag, New York, 1976. Graduate Texts in Mathematics, No. 39.

[2] Sterling K. Berberian. Baer *-rings. Springer-Verlag, New York, 1972. Die Grundlehren der mathematischen Wissenschaften, Band 195.

[3] P. M. Cohn. Free rings and their relations, volume 19 of London Mathematical Society Monographs. Academic Press Inc. [Harcourt Brace Jovanovich Publishers], London, second edition, 1985.

[4] Józef Dodziuk, Peter Linnell, Varghese Mathai, Thomas Schick, and Stuart Yates. Approximating $L^{2}$-invariants and the Atiyah conjecture. Comm. Pure Appl. Math., 56(7):839-873, 2003. Dedicated to the memory of Jürgen K. Moser.

[5] K. R. Goodearl. von Neumann regular rings. Robert E. Krieger Publishing Co. Inc., Malabar, FL, second edition, 1991.

[6] Peter A. Linnell. Division rings and group von Neumann algebras. Forum Math., 5(6):561576, 1993.

[7] Peter A. Linnell. Noncommutative localization in group rings. In Non-commutative localization in algebra and topology, volume 330 of London Math. Soc. Lecture Note Ser., pages 40-59. Cambridge Univ. Press, Cambridge, 2006.

[8] Peter A. Linnell. Embedding group algebras into finite von Neumann regular rings. In Modules and comodules. Proceedings of the international conference, Porto, Portugal, September 6-8, 2006. Dedicated to Robert Wisbauer on the occasion of his 65th birthday, Trends in Mathematics, pages 295-300. Birkhäuser Verlag, Basel, 2008.

[9] Wolfgang Lück. $L^{2}$-invariants of regular coverings of compact manifolds and CW-complexes. In Handbook of geometric topology, pages 735-817. North-Holland, Amsterdam, 2002.

[10] Wolfgang Lück. $L^{2}$-invariants: theory and applications to geometry and K-theory, volume 44 of Ergebnisse der Mathematik und ihrer Grenzgebiete. 3. Folge. A Series of Modern Surveys in Mathematics [Results in Mathematics and Related Areas. 3rd Series. A Series of Modern Surveys in Mathematics]. Springer-Verlag, Berlin, 2002.

[11] J. C. McConnell and J. C. Robson. Noncommutative Noetherian rings. Pure and Applied Mathematics (New York). John Wiley \& Sons Ltd., Chichester, 1987. With the cooperation of L. W. Small, A Wiley-Interscience Publication.

[12] Donald S. Passman. Infinite group rings. Marcel Dekker Inc., New York, 1971. Pure and Applied Mathematics, 6.

[13] Thomas Schick. Integrality of $L^{2}$-Betti numbers. Math. Ann., 317(4):727-750, 2000.

[14] Thomas Schick. Erratum: "Integrality of $L^{2}$-Betti numbers". Math. Ann., 322(2):421-422, 2002.

Peter A. Linnell

Department of Mathematics

Virginia Tech

Blacksburg, VA 24061-0123 
USA

E-mail: plinnell@math.vt.edu

http://www.math.vt.edu/people/plinnell/

Thomas Schick

Mathematisches Institut

Georg-August-Universität Göttingen

Bunsenstr. 3

D-37073 Göttingen

Germany

Email: schick@uni-math.gwdg.de

http://www.uni-math.gwdg.de/schick/ 